\title{
Colaboración con universidades y desempeño innovador en la industria farmacéutica española (2003-2005)
}

\author{
Pablo D'Estea , Josep A. Tribób y Antonio García-Romeroc \\ Instituto de Innovación y Gestión del Conocimiento (CSIC-UPV). Ciudad Politécnica de la Innovación. Valencia. \\ - Departamento de Economía de la Empresa. Universidad Carlos III. Madrid. \\ UPIB. Agencia Laín Entralgo y Departamento de Economia. Universidad Europea de Madrid. Madrid. España.
}

FUNDAMENTO Y OBJETIVOS: Se estudia el efecto en la innovación farmacéutica de la cooperación entre universidades y empresas farmacéuticas en España. De modo análogo, se considera el efecto que tiene la universidad como fuente de información para la innovación en medicamentos.

MATERIAL Y MÉTODO: Se emplean datos procedentes del panel de innovación tecnológica (PITEC) elaborado a partir de las encuestas de innovación de los años 2003 a 2005. Para un total de 132 empresas, se dispone de información de su actividad innovadora (cuatro indicadores), la cooperación con universidades, así como la utilidad del conocimiento que éstas generan. También se emplean variables de control como el tamaño del gasto en I+D o la actividad en biotecnología.

RESULTADOS: El efecto de la cooperación con universidades es positivo y significativo $(p<0,01)$. Además se observa un efecto positivo de la $I+D$ en biotecnología. De modo similar, el efecto de la universidad como fuente de información para la innovación es igualmente positivo $(p<0,01)$. CONCLUSIONES: Las empresas farmacéuticas que han cooperado con universidades tienen una mayor probabilidad de solicitar patentes, así como de que éstas les sean concedidas. Además, la proporción de ventas de productos nuevos es sensiblemente mayor en las empresas que han cooperado con universidades que en las que no lo hacen:

Palabras clave: Innovación. Industria farmacéutica. Cooperación con universidades.

Collaboration with universities and innovate activity in the Spanish pharmaceutical industry (2003-2005)

BACKGROUND AND OBJECTIVES: This article examines, on the one hand, the impact of the cooperation between universities and industry on the performance of innovative activities conducted by pharmaceutical firms in Spain and, on the other hand, the article assesses the impact of universities as a source of information for the innovative activities of the Spanish pharmaceutical industry.

MATERIAL AND METHOD: Data were drawn from the panel of technological innovation, based on the innovation surveys from 2003 to 2005 . The survey included 132 pharmaceutical firms and information was available on the firms' innovative outputs (four indicators), cooperation with universities, and the firms' assessment of the importance of universities as an information source for innovative activities. Firm size, R\&D expenditures and the use of biotechnology were used as control variables. RESULTS: The impact of cooperation with universities was found to be positive and significant $(\mathrm{p}>0.01)$. Moreover, the results show a positive impact of both the use of biotechnology and the use of universities as a source of information on firms' innovative performance $(p<0.01)$. CONCLUSIONS: Pharmaceutical firms that cooperate with universities are more likely to apply for patents and to be granted them. Moreover, the share of sales from new products is larger among firms that have cooperated with universities than among those that have not.

Key words: Innovation. Pharmaceutical industry. Cooperation with universities.

Correspondencia: Dr. A. García-Romero

Agencia Lain Entralgo. Comunidad de Madrid.

Gran Vía 27. 28013 Madrid. España.

Correo electrónico: agr33@salud.madrid.org

\section{Introducción}

La industria farmacéutica contribuye de modo significativo al avance social y económico de los países mediante el desarrollo de medicamentos más eficaces en la lucha contra las enfermedades. Como consecuencia de estos avances tecnológicos, se ha venido observando un aumento significativo tanto de la esperanza como de la calidad de vida, lo que redunda en un importante efecto económico ${ }^{1}$

Para hacer llegar al mercado nuevos medicamentos, las empresas farmacéuticas han de realizar una elevada inversión en 1+D. De hecho, el sector farmacéutico es el sector industrial que más invierte en investigación ${ }^{2}$. En el año 2006 las empresas de este sector destinaron a I+D 844 millones de euros, lo que representa el $18,8 \%$ del gasto total de toda la industria española. Se trata, por lo tanto, de un sector estratégico para la consecución de los objetivos marcados en la estrategia de Lisboa, así como los del Programa Ingenio 201.02,3

En la actualidad, el sector farmacéutico se encuentra ante una encrucijada, dado que, pese al continuo incremento del gasto en I+D en la última década, los resultados en la obtención de nuevos medicamentos no han evolucionado al mismo ritmo ${ }^{3}$. Al mismo tiempo, otros países como China e India se han incorporado con fuerza al escenario de la investigación biomédica incrementando notablemente su captación de fondos privados para desarrollar nuevos fármacos, en detrimento de Europa.

Con objeto de hacer frente a esta situación, en 2007 se puso en marcha la iniciativa de medicamentos innovadores (IMI) ${ }^{4}$, cuya misión principal es favorecer la innovación farmacéutica en Europa. Para alcanzar este objetivo, una de las principales líneas de actuación será el incremento de la colaboración entre empresas y organismos públicos de investigación (OPI)

En relación con la colaboración entre empresas farmacéuticas y $\mathrm{OPI}$, conviene tener en cuenta tres aspectos importantes. En primer lugar, los resultados de la investigación en el seno de los OPI han contribuido de manera fundamental a sentar las bases para el descubrimiento y posterior desarrollo de nuevos fármacos ${ }^{5}$. Tal y como ponen de manifiesto algunos estudios realizados en otros países ${ }^{6}$, la industria farmacéutica está entre las que más emplean los resultados de la investigación científica procedente de universidades y centros públicos de investigación en sus proyectos de I+D. Además, este papel de los OPI en la innovación farmacéutica es cada vez más importante, puesto que el número de medicamentos procedentes de descubrimientos desarrollados en instituciones académicas ha ido creciendo de modo significativo en los últimos años ${ }^{5}$

En segundo lugar, los resultados de la actividad de investigación de los OPI (universidades, hospitales, CSIC, etc.) pueden contribuir a lo largo de todo el proceso de desarrollo de un medicamento ${ }^{8}$. Desde el descubrimiento de principios 
activos hasta los ensayos clínicos, pasando por los estudios de toxicología. Podría decirse, por lo tanto, que sin la participación de los OPI las empresas farmacéuticas no tendrían la misma efectividad obteniendo medicamentos novedosos. En tercer lugar, la difusión del conocimiento generado por los OPI hacia las empresas farmacéuticas tiene lugar por múltiples vías. Entre ellas cabe destacar, por un lado, la colaboración directa entre instituciones académicas y empresas y, por otro, el empleo de los resultados de la investigación realizada por los OPI, a través de publicaciones científicas o nuevos instrumentos o técnicas de investigación. Por supuesto, estas vías de difusión pueden emplearse simultáneamente.

Por otro lado, la colaboración tiene unas características específicas que la hacen especialmente interesante como canal de transferencia de conocimiento. En primer lugar, la colaboración entre los OPI y las empresas permite un intercambio de información en las dos direcciones, en la que a menudo son los progresos en la fase de desarrollo lo que inspira avances en investigación básica. Además, la interacción directa con la comunidad científica permite a las empresas posicionarse favorablemente para identificar oportunidades de investigación derivadas de los avances en la frontera de la ciencia $^{5}$

En este contexto, el presente estudio pretende identificar en qué medida la colaboración con universidades es un factor determinante del output innovador de las empresas farmacéuticas españolas. Con ello se espera tener una mejor comprensión respecto al grado en que el gasto público en investigación contribuye a generar un conocimiento que la industria farmacéutica española emplea de una forma efectiva.

\section{Material y método}

\section{Sujetos de estudio}

Los datos utilizados en este estudio provienen del Panel de Innovación Tecnológica (PITEC) para el periodo 2003-20059. Ese panel se elabora a partir de la encuesta de innovación que realiza anualmente el Instituto Nacional de Estadística (INE). La encuesta proporciona información sobre el proceso innovador y permite observar las relaciones de dicho proceso con aspectos como la estrategia, los factores que favorecen o dificultan la innovación o el impacto económico de la innovación.

La encuesta va dirigida a las empresas de todos los sectores de 10 o más asalariados cuya actividad económica principal se corresponda con las secciones $\mathrm{C}$ a $\mathrm{O}$ de la Clasificación Nacional de Actividades Económicas (CNAE). Las empresas incluidas en el censo (43.000) son las que cumplan al menos uno de los requisitos siguientes: a) empresas que pueden desarrollar actividades de $I+D ; b)$ todas las empresas de más de 200 empleados, y c) una muestra aleatoria extraída del Directorio Central de Empresas (DIRCE).

En el presente estudio se emplea una muestra de $132 \mathrm{em}$ presas farmacéuticas que participaron en las encuestas de 2003 a 2005. El uso de un conjunto de datos longitudinales (datos de panel) permite hacer inferencias acerca de la causalidad de las variables explicativas con relación a la innovación. Ésta es precisamente una de las principales aportaciones del estudio.

\section{Encuesta}

El cuestionario se elabora a partir del Manual de Oslo de la $\mathrm{OCDE}^{10}$ y contiene preguntas sobre distintas facetas de la actividad innovadora en las empresas, así como sus caracte- rísticas. En primer lugar, hay preguntas orientadas a medir la actividad innovadora de las empresas: a) el porcentaje de ventas debido a productos nuevos para la empresa entre $\mathrm{t}-2$ y t (productos nuevos); b) pregunta tipo sí/no que indica si se han solicitado patentes (solicitud patentes); c) pregunta tipo sí/no que indica si se han concedido patentes nuevas (patentes concedidas), y d) pregunta tipo sí/no que indica la existencia de innovaciones de proceso (innovación). Estas variables se emplean como variables dependientes en el análisis multivariable.

En segundo lugar, hay otras preguntas sobre la cooperación en I+D y las fuentes de información empleadas por las empresas: a) pregunta tipo sí/no que indica si la empresa coopera en $1+D$ con universidades $u$ otras entidades (cooperación universidad), b) pregunta tipo sí/no que indica si la empresa emplea una determinada fuente de información, y c) pregunta tipo Likert (alto, medio y bajo) que valora cada una de las fuentes (fuente universidad). Estas variables se utilizan como variables explicativas en el análisis multivariable.

Finalmente, la encuesta incluye preguntas sobre determinadas características de las empresas: a) porcentaje de las ventas destinado a gastos internos en $1+D$ (gastos internos $1+D)$; b) tamaño de la empresa, tomado como el logaritmo de la cifra de negocios (tamaño); c) variable binaria que refleja si se desarrollan actividades de $1+D$ relacionadas con la biotecnología (biotecnología) y d) variable binaria que refleja si la empresa es o no multinacional (multinacional). Estas variables se utilizan como variables de control en el análisis multivariable.

El método de recogida de la información es un sistema mixto que comprende la realización de envíos por correo y la participación de entrevistadores, con un importante apoyo telefónico en la recogida.

\section{Análisis de los datos}

Los datos se analizaron estadísticamente con el programa STATA 10. Las variables continuas se describen mediante media \pm desviación estándar y las categóricas, mediante porcentajes. El análisis se realiza utilizando técnicas de regresión multivariable. En concreto, se plantean varios modelos multivariables que tratan de explicar la influencia que tiene la cooperación con universidades, así como la información que éstas generan sobre el output innovador (medido en sus diversas formas). Estos modelos se estimaron mediante regresiones robustas o a través de modelos probit cuando las variables son dicotómicas. El nivel de significación de parámetros y estadísticos se establece para un valor de $p<0,05$. Es importante indicar que, para evitar problemas de solapamiento entre la variable dependiente (que en algunos casos recoge información desde $\mathrm{t}-2$ hasta $\mathrm{t}$ ) y las variables explicativas, hemos adelantado las variables dependientes uno o dos periodos. Esto, además, permite analizar con un mayor rigor relaciones de causalidad, así como la dinámica de las relaciones encontradas con el paso del tiempo.

\section{Resultados}

Rasgos generales de las empresas farmacéuticas españolas

La tabla 1 presenta las características principales de las empresas de la muestra. El número medio de empleados es de 273 , mientras que las ventas alcanzan un valor de 99,9 millones de euros. En relación con la I+D, el $88 \%$ desarrolla actividades de $1+D$ interna e invierte un promedio del $9,4 \%$ del total de ventas. Asimismo, el $27 \%$ de las empresas desarrolla actividades de $1+D$ relacionadas con la biotecnología. 
D'ESTE P ET AL. COLABORACIÓN CON UNIVERSIDADES Y DESEMPEÑO INNOVADOR EN LA INDUSTRIA FARMACÉUTICA ESPAÑOLA (2003-2005)

TABLA 1

Descripción de la muestra $(n=132)$

\begin{tabular}{|c|c|c|c|}
\hline & $\begin{array}{c}\text { Total }(n=132) \\
\text { media } \pm \mathrm{DE}\end{array}$ & $\begin{array}{l}\text { Nacionales } \\
(n=82), \text { media }\end{array}$ & $\begin{array}{l}\text { Multinacionales } \\
(n=50) \text {, media }\end{array}$ \\
\hline $\begin{array}{l}\text { Tamaño (empleados), n } \\
\text { Tamaño (ventas en miliones de euros) } \\
\text { I+D interna (sí/no) (\%) } \\
\text { Gasto en I+D/ventas (\%) } \\
\text { Actividad en biotecnología (sí/no) }(\%) \\
\text { Introducción de nuevo producto (síno) (\%) } \\
\text { Introducción de nuevo proceso (si/no) }(\%) \\
\text { Nuevos productos para la empresa representan más del } 10 \% \text { de sus ventas (\%) } \\
\text { Novedades representan más del } 10 \% \text { de las ventas } \\
\text { Patentes solicitadas (sí/no) (\%) } \\
\text { Patentes solicitadas en EPO O USPTO (sí/no) }(\%)\end{array}$ & $\begin{array}{l}273 \pm 310,6 \\
99,9 \pm 163,7 \\
87,9 \pm 32,8 \\
9,42 \pm 16,8 \\
26,5 \pm 44,3 \\
64,4 \pm 48,1 \\
66,6 \pm 47,3 \\
26,5 \pm 44,3 \\
18,2 \pm 38,7 \\
33,3 \pm 47,3 \\
16,7 \pm 37,4\end{array}$ & $\begin{array}{r}163,0 \\
41,9 \\
91,5 \\
10,3 \\
25,6 \\
62,2 \\
66,7 \\
28,0 \\
12,2 \\
36,6 \\
15,9\end{array}$ & $\begin{array}{r}454,0 \\
195,1 \\
82,0 \\
8,0 \\
28,0 \\
68,0 \\
66,6 \\
24,0 \\
28,0 \\
28,0 \\
18,0\end{array}$ \\
\hline
\end{tabular}

Fuente: PITEC y elaboración propia.

Respecto al tipo de empresas, el $62 \%$ son nacionales, frente al $38 \%$ de empresas multinacionales. Entre las primeras hay una mayor proporción de empresas que desarrollan actividades de $1+D(91,5 \%)$ y además invierten en este concepto un porcentaje de las ventas superior al de las empresas nacionales. Resulta llamativo, sin embargo, que sean las nacionales donde hay una mayor proporción (28\%) de empresas con actividades en biotecnología.

En lo que respecta a la innovación, un $64 \%$ del total de empresas señala haber introducido un nuevo producto durante el periodo 2003-2005, que es ligeramente superior entre las multinacionales. Este resultado no resultó significativo en el análisis multivariable posterior. La repercusión de estas innovaciones en el total de ventas no es demasiado alta, yá que sólo el $18 \%$ de las empresas indica que los productos nuevos introducidos por la empresa representan más del 10\% de su cifra de ventas. En este caso sí resulta significativa la diferencia entre empresas nacionales $(12 \%)$ y multinacionales $(28 \%)$.

En cuanto a la solicitud de patentes, el 33\% de las empresas to han hecho en el periodo 2003-2005; el porcentaje observado en las empresas nacionales es sensiblemente superior, resultado que se confirma en el análisis multivariable posterior. Esto podría explicarse por la tendencia de las multinacionales a solicitar patentes desde sus sedes centrales. El porcentaje de empresas que solicitan patentes se reduce al $17 \%$ cuando se considera únicamente las patentes solicitadas en la EPO o en la USPTO.

\section{Cooperación y fuentes de información externas}

Las empresas farmacéuticas se muestran muy activas en el establecimiento de acuerdos de cooperación para la innovación con otras empresas o entidades. De manera más específica, el $44 \%$ de las empresas farmacéuticas indica haber colaborado durante el período 2003-2005 con al menos una de las entidades que se presentan en la tabla 2. Según estos datos, las universidades constituyen el principal socio con el que las empresas farmacéuticas establecen acuerdos de cooperación en actividades de innovación. En particular, el $30 \%$ de las empresas farmacéuticas ha cooperado con universidades en actividades de $1+D$ e innovación, mientras que esta proporción se reduce al $12 \%$ para las empresas en otros sectores. Los datos que se presentan en la tabla 3 concuerdan con los resultados de otros estu$\operatorname{dios}^{6,11-13}$.

Por su parte, en lo que concierne a las fuentes de información externas, las empresas farmacéuticas valoran en mayor medida estas fuentes para sus actividades de innovación. En particular, la tabla 3 pone de manifiesto que más
TABLA 2

Cooperación en innovación con distintos tipos de socios (2003-2005)

\begin{tabular}{|l|c|c|}
\hline & \multicolumn{2}{|c|}{ Empresas (\%) } \\
\hline & $\begin{array}{c}\text { Industria } \\
\text { farmacéutica }\end{array}$ & $\begin{array}{c}\text { Todos los } \\
\text { Todos los sectores }\end{array}$ \\
\hline Otras empresas del mismo grupo & 19,7 & 4,4 \\
Proveedores & 13,6 & 2,1 \\
Clientes & 12,1 & 3,8 \\
Competidores & 10,6 & 1,9 \\
Consultores, lab. comerciales & 23,5 & 6,9 \\
Universidades & 30,3 & 11,9 \\
Organismos públicos de investigación & 21,2 & 8,1 \\
Centros tecnológicos & 10,6 & 2,9 \\
\hline
\end{tabular}

Fuente: PITEC y elaboración propia.

del $80 \%$ de las empresas del sector farmacéutico emplea las fuentes de información que se incluyen explícitamente en la encuesta de innovación. Dicha proporción es particu larmente alta en el caso del empleo de las universidades como fuente externa de información. El $84 \%$ de las empresas farmacéuticas destaca haber empleado las universidades como fuente externa de información, mientras que son el $51 \%$ del conjunto de los sectores considerados en la encuesta de innovación. Asimismo, las empresas farmacéuticas que valoran la universidad como una fuente de «elevada importancia» suponen el $28 \%$, frente al $18 \%$ del total de sectores. En definitiva, las universidades son la fuente externa de conocimiento de mayor importancia para las em. presas farmacéuticas, por delante de proveedores, clientes o competidores. Estos datos concuerdan con los observados en otros países ${ }^{7}$

\section{Cooperación e innovación. Análisis multivariable}

Los resultados para el efecto de la cooperación con universidades sobre la innovación se presentan en la tabla 4 , que está organizada del modo siguiente: las primeras dos columnas corresponden a la variable dependiente productos nuevos; las dos siguientes se emplean para la solicitud de patentes; las columnas 5 y 6 recogen los resultados para la concesión de patentes y, por último, las dos columnas finales muestran los resultados para la variable dicotómica que recoge la innovación de proceso. Asimismo, las columnas impares presentan los resultados para la variable dependiente adelantada un periodo $(t+1)$ y en las columnas pares, la variable adelantada dos periodos $(t+2)$. Con ello se pretende observar la evolución del efecto de la cooperación con universidades en las diversas variables 
TABLA 3

Fuentes externas de información de la industria farmacéutica (2003-2005)

\begin{tabular}{|c|c|c|c|c|}
\hline \multirow{3}{*}{ Fuentes de información } & \multicolumn{4}{|c|}{ Empresas (\%) } \\
\hline & \multicolumn{2}{|c|}{ Emplean cada fuente } & \multicolumn{2}{|c|}{ Valoran cada fuente como muy importante } \\
\hline & Farmacéutica & Total & Farmacéutica & Total \\
\hline $\begin{array}{l}\text { Proveedores } \\
\text { Clientes } \\
\text { Competidores } \\
\text { Consultores } \\
\text { Universidades } \\
\text { Organismos públicos de investigación } \\
\text { Centros tecnológicos } \\
\text { Conferencias, ferias, etc. } \\
\text { Revistas, publicaciones } \\
\text { Asociaciones profesionales }\end{array}$ & $\begin{array}{l}81,5 \\
85,5 \\
83,1 \\
80,6 \\
83,9 \\
70,2 \\
62,9 \\
81,5 \\
83,1 \\
73,4\end{array}$ & $\begin{array}{l}47,9 \\
50,8 \\
49,8 \\
50 \\
51,5 \\
43,7 \\
39,4 \\
49,8 \\
52,1 \\
44\end{array}$ & $\begin{array}{r}9,7 \\
20,2 \\
10,5 \\
17,7 \\
28,2 \\
17,7 \\
9,7 \\
10,5 \\
17,7 \\
5,6\end{array}$ & $\begin{array}{r}6,7 \\
12,1 \\
7,7 \\
11,3 \\
17,9 \\
10 \\
5,6 \\
7,3 \\
12,3 \\
4,4\end{array}$ \\
\hline
\end{tabular}

Fuente: PITEC y elaboración propia.

\section{TABLA 4}

Efecto de la cooperación con universidades en la innovación

\begin{tabular}{|c|c|c|c|c|c|c|c|c|}
\hline Variable dependiente & $\begin{array}{c}\text { Productos } \\
\text { nuevos } \\
(t+1)\end{array}$ & $\begin{array}{c}\text { Productos } \\
\text { nuevos } \\
(t+2)\end{array}$ & $\begin{array}{l}\text { Solicitud } \\
\text { patentes } \\
\text { (t) }\end{array}$ & $\begin{array}{c}\text { Solicitud } \\
\text { patentes } \\
(t+1)^{\mathrm{a}}\end{array}$ & $\begin{array}{c}\text { Patentes } \\
\text { concedidas } \\
(t+1)\end{array}$ & $\begin{array}{c}\text { Patentes } \\
\text { concedidas } \\
(\mathrm{t}+2)\end{array}$ & $\begin{array}{c}\text { Innovación } \\
(t+1)\end{array}$ & $\begin{array}{l}\text { Innovación } \\
\qquad(t+2)\end{array}$ \\
\hline $\begin{array}{l}\text { Cooperación con universidad } \\
\text { Gastos internos en } 1+D \\
\text { Tamaño } \\
\text { Biotecnología } \\
\text { Multinacional } \\
\text { Constante } \\
\text { F (p)" } \\
\text { Casos, n }\end{array}$ & $\begin{array}{c}0,09 \\
0,01 \\
0,02^{c} \\
0,12^{b} \\
-0,11^{b} \\
-0,30 \\
2,74^{b} \\
289\end{array}$ & $\begin{array}{c}0,23^{b} \\
0,01 \\
0,02 \\
0,22^{b} \\
-0,12 \\
-0,38 \\
4,85^{b} \\
132\end{array}$ & $\begin{array}{c}0,40^{\mathrm{c}} \\
0,02^{\mathrm{d}} \\
0,13^{\mathrm{b}} \\
0,70^{\mathrm{b}} \\
-0,30^{\mathrm{c}} \\
-2,68^{\mathrm{b}} \\
8,87^{\mathrm{b}} \\
479\end{array}$ & $\begin{array}{c}0,41^{\mathrm{c}} \\
0,01 \\
0,14^{\mathrm{b}} \\
0,85^{\mathrm{b}} \\
-0,41^{\mathrm{c}} \\
-2,94^{\circ} \\
9,87^{\mathrm{b}} \\
289\end{array}$ & $\begin{array}{c}0,06 \\
0,01 \\
0,14^{\mathrm{b}} \\
0,68^{\mathrm{b}} \\
-0,19 \\
-3,89^{\mathrm{b}} \\
58,84^{\mathrm{b}} \\
289\end{array}$ & $\begin{array}{c}0,83^{\mathrm{b}} \\
0,01^{\mathrm{d}} \\
0,14^{\mathrm{b}} \\
0,77^{\mathrm{b}} \\
\cdots 0,16 \\
-4,50^{\mathrm{b}} \\
39,05^{\mathrm{b}} \\
132\end{array}$ & $\begin{array}{c}0,61^{b} \\
0,01^{b} \\
0,01 \\
0,29^{c} \\
-0,05 \\
0,01 \\
29,39^{b} \\
289\end{array}$ & $\begin{array}{c}0,42^{\mathrm{c}} \\
0,01^{\mathrm{c}} \\
0,01 \\
0,38 \mathrm{c} \\
0,03 \\
-0,27 \\
12,20^{\mathrm{b}} \\
132\end{array}$ \\
\hline
\end{tabular}

Se consideran la variables contemporánea (t) y adelantada un periodo $(t+1)$ para definir û́n periodo de diferencia con la concesión de patentes.

$p<0,01$.

$p<0.05$

En el caso de las estimaciones probit, el test de bondad de ajuste es el LR test.

de output innovador. Hay que indicar que las 132 observaciones con las que se ha realizado el análisis descriptivo corresponden a las estimaciones en las que la variable dependiente está adelantada dos periodos $(t+2)$ y, por 10 tanto, hay información para los tres años considerados en este estudio.

La tabla 4 indica que la cooperación con universidades tiene un efecto positivo en el porcentaje de ventas debido a productos nuevos para la empresa. Este efecto resulta significativo cuando la variable dependiente se adelanta dos periodos, ya que en ese caso recoge las ventas en nuevos productos desde t hasta $t+2$, y de esta forma se evitan solapamientos con las variables explicativas. Éste es un resultado robusto, puesto que se mantiene para las diversas variables dicotómicas de output innovador: solicitud de patentes, concesión de patentes e innovaciones de proceso.

Respecto de las variables de control, se observa que las empresas más grandes y las relacionadas con la biotecnología son las que muestran unos mayores niveles de innovación, particularmente cuando se considera como indicadores la solicitud y la concesión de patentes. En cuanto al carácter multinacional de las empresas, los resultados parecen indicar que es menos probable que soliciten una patente en comparación con las empresas nacionales. Probablemente esto tenga que ver con que la solicitud de patentes por empresas multinacionales la realizan las empresas matrices y fuera de España. Por último, cabe indicar que la intensidad en el gasto interno en $1+D$ no siempre tiene un efecto positivo en el output innovador.
En cuanto al efecto que tiene la universidad como fuente de información, la tabla 5 presenta los resultados siguiendo la misma estructura que la tabla 4.

Los resultados ponen de manifiesto que el conocimiento generado en las universidades tiene un efecto positivo en la innovación farmacéutica para cualquier indicador y periodo. Además, se observa que, en general, los coeficientes son mayores para la variable fuente que para la variable coope. ración (tabla 5 ).

Respecto de las variables de control, los resultados son coherentes con los obtenidos para la cooperación. Las empresas más grandes, especialmente si no son multinacionales, y que desarrollan actividades de biotecnología son las que obtienen mejores resultados innovadores. Asimismo, los gastos internos en $1+D$ tienen un efecto relativo en la innovación en las empresas de la muestra.

En este contexto, y a la vista de los resultados anteriores, tiene interés plantearse la relación que existe entre las variables cooperación y fuente. Hemos realizado una estimación de la variable fuente en función de la variable cooperación, incorporando los controles definidos en las tablas 4 y 5 , y la relación que se obtiene es positiva y significativa $(p<0,1)$, lo que podría interpretarse del modo siguiente. Cuando las empresas cooperan con universidades, desarrollan las habilidades necesarias para absorber el conocimiento que proviene de las universidades en los años posteriores a la colaboración. Esto refuerza el efecto positivo que tiene la cooperación con universidades en la innovación farmacéutica. Este efecto ligado a la universidad como fuente de información permite especular sobre el impacto duradero que tiene la coopera- 
D'ESTE P ET AL. COLABORACIÓN CON UNIVERSIDADES Y DESEMPEÑO INNOVADOR EN LA INDUSTRIA FARMACÉUTICA ESPAÑOLA (2003-2O05)

TABLA 5

Efecto de la información procedente de las universidades en la innovación

\begin{tabular}{|c|c|c|c|c|c|c|c|c|}
\hline Variable dependiente & $\begin{array}{c}\text { Productos } \\
\text { nuevos } \\
(t+1)\end{array}$ & $\begin{array}{c}\text { Productos } \\
\text { nuevos } \\
(t+2)\end{array}$ & $\begin{array}{l}\text { Solicitud } \\
\text { patentes } \\
\text { (t) }\end{array}$ & $\begin{array}{c}\text { Patentes } \\
\text { concedidas } \\
(t+1)^{\mathrm{a}}\end{array}$ & $\begin{array}{c}\text { Patentes } \\
\text { concedidas } \\
(t+1)\end{array}$ & $\begin{array}{c}\text { Concesión } \\
\text { patentes } \\
(\mathrm{t}+2)\end{array}$ & $\begin{array}{l}\text { Innovación } \\
\qquad(t+1)\end{array}$ & $\begin{array}{l}\text { Innovación } \\
\quad(t+2)\end{array}$ \\
\hline $\begin{array}{l}\text { Fuente universidad } \\
\text { Gastos internos en } 1+D \\
\text { Tamaño } \\
\text { Biotecnología } \\
\text { Multinacional } \\
\text { Constante } \\
\mathrm{F}(p)^{\mathrm{e}} \\
\text { Casos, } \mathrm{n}\end{array}$ & $\begin{array}{c}14,59^{\mathrm{b}} \\
0,04 \\
-0,72^{\mathrm{j}} \\
1,38 \\
3,86 \\
15,18 \\
0,13^{\mathrm{b}} \\
289\end{array}$ & $\begin{array}{c}14,65 \\
0,09 \\
-0,14 \\
-0,08 \\
5,70 \\
1,64 \\
0,03^{\circ} \\
132\end{array}$ & $\begin{array}{c}0,85^{c} \\
0,01 \\
0,13^{c} \\
0,67^{c} \\
-0,266^{\circ} \\
-3,51^{\circ} \\
71,64^{c} \\
479\end{array}$ & $\begin{array}{c}0,84^{c} \\
0,01 \\
0,15^{c} \\
0,78^{c} \\
-0,46^{c} \\
-3,73^{c} \\
82,28^{c} \\
289\end{array}$ & $\begin{array}{c}0,63^{c} \\
0,01^{1} \\
0,14 \\
0,65 \\
-0,13 \\
-4,34 \\
65,67 \\
289\end{array}$ & $\begin{array}{c}0,64^{\circ} \\
0,01^{\circ} \\
0,17^{\circ} \\
0,78^{c} \\
-0,14 \\
-4,53^{c} \\
34,80^{c} \\
132\end{array}$ & $\begin{array}{c}1,57^{i} \\
0,01 \\
-0,01 \\
0,26^{5} \\
-0,02 \\
-0,97 \\
45,13 \\
289\end{array}$ & $\begin{array}{c}1,12 \\
0,01 \\
0,01 \\
0,35 \\
0,05 \\
0,06 \\
15,90 \\
132\end{array}$ \\
\hline
\end{tabular}

aSe consideran la variables contemporánea ( $t$ ) y adelantada un periodo $(t+1)$ para definir un periodo de diferencia con la concesión de patentes.

${ }^{5} p<0,05$.

$p<0,01$

'p $p<0,1$

EEn el caso de las estimáciones probit, el test de bondad de ajuste es el LR test.

ción con universidades en la innovación de las empresas farmacéuticas.

\section{Conclusiones}

Los resultados obtenidos en este trabajo ponen de manifiesto la relevancia de las universidades en la innovación farmacéutica, bien mediante colaboración directa en I+D, bien a través de la absorción del conocimiento que se genera en las universidades. En particular, el efecto es importante en el porcentaje de ventas de nuevos productos, en la solicitud y concesión de patentes, así como en la probabilidad de generar innovaciones de proceso. Por lo tanto, puede concluirse que la investigación generada en el entorno académico tiene un importante efecto económico en las empresas del sector farmacéutico español. Estos resultados son similares a los observados en otros países ${ }^{5}$.

En particular, los resultados obtenidos en este estudio muestran que las empresas farmacéuticas que colaboran con la universidad en proyectos de investigación son 1,5 veces más proclives a solicitar patentes y a introducir innovaciones de proceso que las empresas que no colaboran con las universidades. Y son 2,3 veces más proclives a obtener patentes que las empresas que no cooperan con universidades. Nuestros resultados también muestran que la intensidad de gasto in $1+D$ no siempre tiene un impacto positivo y significativo en la innovación. Esto puede reflejar que para la consecución del éxito en ventas tan importante como la I+D puede ser el esfuerzo en marketing que realizan las empresas o que la solicitud de patentes está más asociada con la escala de gasto en $1+D$ más que la intensidad de la $I+D$, habida a cuenta del claro efecto positivo de la variable tamaño.

A su vez, al estudiar el efecto diferencial de la presencia de multinacionales, hemos comprobado que las empresas no multinacionales son las que generan un mayor output innovador. Podemos especular que una interacción más intensa de las empresas multinacionales con las universidades mejoraría el output innovador de éstas.

Finalmente, se ha comprobado que las empresas que cooperan con universidades tienen mayor capacidad para aprovechar los conocimientos generados en el entorno académico. Esta circunstancia refuerza el efecto positivo que la cooperación con universidades tiene en la innovación en las empresas. Estudiar con mayor profundidad este efecto será objeto de investigaciones futuras.

\section{Declaración de conflicto de intereses}

Los autores han declarado no tener ningún conflicto de intereses.

\section{Acrónimos}

EPO: European Patent Office.

USPTO: United States Patent and Trademark Office.

.

\section{REFERENCIAS BIBLIOGRÁFICAS}

1. Lichtenberg F. Pharmaceutical innovation, mortality reduction, and economic growth. En: Murphy KM, Topel RH, editores. Measuring the gains from medical research: an economic approach. Chicago: University of Chicago Press; 2003; p. 74-109.

2. Memoria Anual 2007. Madrid: Farmaindustria; 2007

3. INE. Estadísticas sobre actividades en I+D. Año 2006 (Resultados provi sionales) [citado 1 Dic 2007]. Disponible en: http://www.ine.es/prensa/ np476.pdf

4. Innovative medicines initiative. Creating Biomedical R\&D Leadership for Europe to benefit patients and society [citado 15 May 2008]. Disponible en: http://www.imi-europe.org/Lists//MIPublicationDocuments//MI-GB-research-agenda-006v2-15022008.pdf

5. Cockburn IM, Henderson RM. Absorptive capacity, coauthoring behaviour, and the organization of research in drug discovery. The Journal of Industrial Economics. 1998;XLVI:157-82.

6. Laursen K, Saiter A. Searching high and low: what types of forms use universities as a source of innovation? Research Policy, 2004:33:1201-15.

7. Cohen WM, Nelson RR, Walsh JP. Links and impacts: the influence of public research on industrial R\&D. Management Science. 2002;48:1-23.

8. Toole AA. Does public scientific research complement private investment in research and development in the pharmaceutical industry? Journal of Law and Economics. 2007:50:81-103.

9. SISE. La base de datos panel de innovación tecnológica (PITEC) Icitado 15 Feb 2008]. Disponible en: http://sise.fecyt.es/Estudios/PITEC/basedatosPITEC.pdf

10. OECD. The Osto Manual. Paris: OECD; 1997

11. Tether BS. Who co-operates for innovation, and why. An empirical analysis. Research Policy. 2002;31:947-67.

12. Belderbos $R$, Carree $M$, Lokshin $B$. Cooperative $R \& D$ and firm performance. Research Policy. 2004:33:1477-92

13. Vega Jurado JM. Las estrategias de innovación en la industria manufac turera española: sus determinantes y efectos sobre el desempeño innovador [tesis doctoral]. Valencia: Universidad Pofitécnica de Valencia. 